\title{
Analysis of the Voltage Swing for Logic and Memory Applications in Si/SiGe Resonant Interband Tunnel Diodes Grown by Molecular Beam Epitaxy
}

\author{
Sung-Yong Chung, Student Member, IEEE, Niu Jin, Member, IEEE, Ryan E. Pavlovicz, Ronghua Yu, \\ Paul R. Berger, Senior Member, IEEE, and Phillip E. Thompson, Senior Member, IEEE
}

\begin{abstract}
A method is investigated to directly engineer the voltage swing in SiGe resonant interband tunnel diodes (RITDs). Voltage swing, defined here as the voltage difference between the peak voltage and the projected peak voltage, is independent of series resistance, and thus directly impacts the noise margin in hybrid tunnel diode memory and logic applications. The three components of the total RITD current are analyzed to describe the voltage swing. The dependence of voltage swing on $\delta$-doping concentrations and post-growth annealing temperatures in SiGe RITDs grown by low-temperature molecular beam epitaxy (LT-MBE) is investigated and the experimental results are compared with a theoretical analysis. Techniques to increase the voltage swing are discussed.
\end{abstract}

Index Terms-Annealing, circuit noise, logic circuit fault tolerance, semiconductor device doping, semiconductor epitaxial layers, semiconductor junctions, tunnel diode, tunnel diode circuits.

\section{INTRODUCTION}

$\mathbf{T}$ HE TUNNEL DIODE has remarkable properties due to its folded $I-V$ characteristics which feature both a negative differential resistance (NDR) and an ultra-high-speed transient response. These characteristics are very useful to circuit designers for applications including memory, logic and mixed signals [1]-[4]. In brief, when a tunnel diode is integrated with a transistor or two tunnel diodes are connected in series, the circuit latches to two stable operating points and switching operation

Manuscript received January 22, 2006; revised August 28, 2006. The work of The Ohio State University was supported in part by the National Science Foundation under Grant DMR-0103248 and Grant ECS-0323657. The work at NRL was supported by the Office of Naval Research (ONR). The review of this paper was arranged by Associate Editor D. Frank.

S.-Y. Chung was with the Department of Electrical and Computer Engineering, Ohio State University, Columbus, OH 43210 USA. He is now with Spansion, Inc., Sunnyvale, CA 94088 USA.

N. Jin was with the Department of Electrical and Computer Engineering, Ohio State University, Columbus, OH 43210 USA. He is now with the Micro and Nanotechnology Laboratory, University of Illinois, Urbana-Champaign, Urbana, IL 61801 USA.

R. E. Pavlovicz was with the Department of Electrical and Computer Engineering, Ohio State University, Columbus, OH 43210 USA. He is now with the Department of Biomedical Engineering. Ohio State University, Columbus, $\mathrm{OH}$ 43210 USA.

R. Yu is with the Department of Physics, Ohio State University, Columbus, OH 43210 USA.

P. R. Berger is with the Department of Electrical and Computer Engineering and the Department of Physics, Ohio State University, Columbus, OH 43210 USA (e-mail: pberger@ieee.org).

P. E. Thompson is with the Naval Research Laboratory, Washington, DC 20375 USA.

Digital Object Identifier 10.1109/TNANO.2007.891831 or data saving can be possible between those two points. Furthermore, since the tunneling phenomenon is a majority carrier effect, the speed of circuits incorporating NDR devices can be greatly improved. Many high-performance tunnel diodes have been reported using III-V material systems [5], but the lack of an integration process compatible with complementary metal oxide semiconductor (CMOS) or SiGe heterojunction bipolar transistor (HBT) technology has limited their utility in VLSI technology. Recent work in Si-based tunnel diodes [6]-[10] and their integration with Si-based CMOS [11] and SiGe HBT [12] make these devices promising for future applications.

While peak-to-valley current ratio (PVCR) and peak current density $\left(J_{P}\right)$ are common figure-of-merits used to evaluate DC performance of tunnel diodes, there is another important figure-of-merit that must be considered at the circuit level, namely, the voltage swing. It is defined as the voltage difference between the peak voltage and the projected peak voltage onto the forward biased diffusion current region [see Fig. 2(d)]. Voltage swing is an excellent indicator of the signal-to-noise margin for tunnel diode based memory and logic circuits [13], [14]. Large voltage swings make the separation between two stable operation points wider, resulting in better noise tolerance in logic and memory applications. As defined above, voltage swing, unlike voltage span which is the voltage difference between the peak and valley voltages, measures the voltage difference at the same amount of current, thus it excludes any series resistance effects which can significantly skew the voltage span. For this voltage swing study, Si-based resonant interband tunnel diodes (RITDs), which have been extensively studied by the authors [7], [8] and others [9] were used.

Si-based RITDs have tremendous flexibility to vary $J_{P}$ from $20 \mathrm{~mA} / \mathrm{cm}^{2}$ [15] to $218 \mathrm{kA} / \mathrm{cm}^{2}$ [8]. PVCRs typically range between 3 and 6 [7], [9] by: a) controlling the Ge composition of $\mathrm{Si}_{x} \mathrm{Ge}_{1-x}$ within the intrinsic barrier and each barrier layer's thickness; b) adjusting substrate growth temperature; c) adjusting post-growth annealing conditions; and d) inserting $\mathrm{SiGe}$ cladding layers if boron is used as the p-type $\delta$-doping injector.

In this paper, we discuss a way to engineer the voltage swing, another figure-of-merit of NDR device that is very important in the estimation of the signal-to-noise margin for the tunnel diodes in digital circuit applications. We will show the effects of the $\delta$-doping concentration on peak current, valley current, and the tunnel diode's voltage swing. The magnitude of the $\delta$-doping concentrations of the injectors has been varied for this investigation and different annealing temperatures have been examined. 


\begin{tabular}{|c|c|}
\hline $100 \mathrm{~nm} \mathrm{n}^{+} \mathrm{Si}$ & \\
\hline $2 \mathrm{~nm}$ undoped $\mathrm{Si}$ & \multirow{4}{*}{$\longleftarrow$ B $\delta$-doping plane } \\
\hline $4 \mathrm{~nm}$ undoped $\mathrm{Si}_{0.6} \mathrm{Ge}_{0.4}$ & \\
\hline $1 \mathrm{~nm} \mathrm{p}^{+} \mathrm{Si}_{0.6} \mathrm{Ge}_{0.4}$ & \\
\hline $54 \mathrm{~nm} \mathrm{p}^{+} \mathrm{Si}$ & \\
\hline
\end{tabular}

Fig. 1. Schematic diagram of the SiGe RITD structure. The key differences for each sample studied are the dopant deposition durations for both $\delta$-doped layers, 200, 100, 50, and $25 \mathrm{~s}$, which effectively equates to $\delta$-doped sheet carrier concentrations of $1 \times 10^{14} \mathrm{~cm}^{-2}, 5 \times 10^{13} \mathrm{~cm}^{-2}, 2.5 \times 10^{13} \mathrm{~cm}^{-2}$, and $1.25 \times 10^{13} \mathrm{~cm}^{-2}$, respectively.

From the analysis of the peak and valley current components, a correlation between PVCR and voltage swing is also discussed.

\section{EXPERIMENTAL}

Four $\mathrm{Si} / \mathrm{SiGe}$ RITDs were grown by LT-MBE and their growth conditions and device structures were designed to be identical except for both $\delta$-doped layers described below. During the MBE growth, $\delta$-doped layers are formed by stopping the growth (i.e., closing the $\mathrm{Si}$ and Ge shutters) and depositing only a dopant. The dopant concentration is usually specified by the sheet concentration and expressed in terms of atoms $/ \mathrm{cm}^{2}$. In this paper, we also use the effective bulk dopant concentration of the $\delta$-doped layers, expressed in atoms $/ \mathrm{cm}^{3}$, which is determined using the assumption that the dopant atoms in the as-grown RITD are confined in a layer having a width of $1 \mathrm{~nm}$. The key difference for the $\delta$-doped layer pairs is the duration of the $\delta$-doping deposition, 200, 100, 50, and $25 \mathrm{~s}$, respectively. This variation effectively varied the $\delta$-doped injector sheet carrier concentrations as follows: $1 \times 10^{14} \mathrm{~cm}^{-2}, 5 \times 10^{13} \mathrm{~cm}^{-2}$, $2.5 \times 10^{13} \mathrm{~cm}^{-2}$, and $1.25 \times 10^{13} \mathrm{~cm}^{-2}$, respectively.

The epitaxial growth was carried out in a Vacuum Generators V-80 MBE system using solid sources. The basic layer structure, shown in Fig. 1, has an n-on-p growth sequence. Growths were initiated with a $2 \mathrm{~nm}$ undoped $\mathrm{Si}$ buffer layer grown at $650{ }^{\circ} \mathrm{C}$ on a $\mathrm{Si}(100) \mathrm{p}^{+}(0.015-0.04 \mathrm{ohm}-\mathrm{cm})$ substrate (not shown in Fig. 1). The substrate temperature was lowered to $500{ }^{\circ} \mathrm{C}$ for the growth of a $54 \mathrm{~nm}$ B-doped $\mathrm{p}^{+}-\mathrm{Si}$ buffer layer. After the substrate temperature was reduced to $320^{\circ} \mathrm{C}$, the remaining active device layers were grown. The growth at $320^{\circ} \mathrm{C}$ includes a 1-nm B-doped SiGe, a B $\delta$-doped layer, spacer layers consisting of 4-nm undoped $\mathrm{SiGe}$ and 2-nm undoped Si layers, a P $\delta$-doped layer, and finally a $100-n m$ P-doped $n^{+}$-Si cap layer for ohmic contacts. During the growth, the B and P cell temperatures were kept constant at $1870^{\circ} \mathrm{C}$ and $750{ }^{\circ} \mathrm{C}$, respectively. All growth was carried out at a rate of $0.1 \mathrm{~nm} / \mathrm{s}$.

Prior to device fabrication, samples were rapid thermal annealed (RTA) at a temperature of $750^{\circ} \mathrm{C}$ in a forming gas $(95 \%$ $\mathrm{N}_{2} / 5 \% \mathrm{H}_{2}$ ) ambient using a Modular Process Technology Corporation RTP-600S furnace. In order to study post-growth annealing effects, additional RTA temperatures of $700{ }^{\circ} \mathrm{C}$, $800{ }^{\circ} \mathrm{C}$, and $850^{\circ} \mathrm{C}$ were chosen for three extra samples taken from the wafer with $1 \times 10^{14} \mathrm{~cm}^{-2} \delta$-doped layers. All RTA times were fixed at $60 \mathrm{~s}$. Ti/Au ohmic contacts $(5,18,50$, and $75 \mu \mathrm{m}$ in diameter) were patterned photolithographically using liftoff and electron-beam evaporation, while backside ohmic contacts of $\mathrm{Ti} / \mathrm{Au}$ were made by deposition across the full surface. Using the top contact metal as a self-aligned etch mask, all diodes were formed into a mesa structure approximately $300 \mathrm{~nm}$ tall. The wet etchant for mesa etching was $\mathrm{HF}: \mathrm{H}_{2} \mathrm{O}: \mathrm{HNO}_{3}(1: 100: 100)$ by volume ratio and etch rates varied between $100-150 \mathrm{~nm} / \mathrm{min}$.

\section{RESULTS AND DISCUSSION}

\section{A. Analysis of Key Influences on an Interband Tunnel Diode's Voltage Swing}

The I-V characteristics of forward biased interband tunnel diodes consists of three current components: band-to-band tunneling current, excess current, and thermal diffusion current. In this study, on the voltage swing modulation of RITD, three different cases are considered and analyzed. All three of these cases assume that the band-to-band tunneling current component and thermal diffusion component are constant, shown in Fig. 2. However, the contribution by the excess current is varied here between the following three cases, as follows: 1) thermal diffusion current can be neglected at the projected voltage level which means the excess current dominates [Fig. 2(a) for Case I]; 2) neither thermal diffusion nor excess current can be neglected and both contribute to the projected voltage [Fig. 2(b) for Case II]; and 3) the excess current component is negligible which implies that the thermal diffusion current dominates at the projected peak voltage [in Fig. 2(c) for Case III].

As illustrated in Fig. 2, when the projected peak voltage is determined by the thermal diffusion I-V characteristics only (Case III), the largest voltage swing can be obtained, if other conditions remain unchanged. However, generally for an intrinsic Si-based RITD with zero series resistance, the projected voltage is smaller than $0.7 \mathrm{~V}$ [15], indicative that Case I with significant excess current often dominates. Thus, the I-V relationship of a tunnel diode for the calculation of voltage swing can be written as a combination of the peak tunneling current component and excess current component, neglecting the thermal diffusion current, because the peak voltage is primarily determined by the peak tunneling current and the projected peak voltage is primarily determined by the excess current [16]

$$
\begin{aligned}
I & \cong I_{t}+I_{e x} \\
& =I_{P}\left(\frac{V}{V_{P}}\right) \exp \left(1-\left(\frac{V}{V_{P}}\right)\right)+I_{v} \exp \left[A\left(V-V_{V}\right)\right]
\end{aligned}
$$

where $I_{t}$ and $I_{e x}$ are the tunneling current and excess current components, respectively. $I_{P}$ and $V_{P}$ are the peak current and peak voltage and $I_{V}$ is the valley current at the valley voltage, $V_{v}$. A is a fitting parameter. The definition of the voltage swing, then, can be determined from the following identity:

$$
\begin{aligned}
& I_{V_{P}}-I_{V_{\mathrm{Pr}}}=I_{P}+I_{V} \exp \left[A\left(V_{P}-V_{V}\right)\right] \\
& -\left\{I_{P}\left(\frac{V_{\mathrm{Pr}}}{V_{P}}\right) \exp \left(1-\frac{V_{\mathrm{Pr}}}{V_{P}}\right)+I_{v} \exp \left[A\left(V_{\mathrm{Pr}}-V_{V}\right)\right]\right\}=0 .
\end{aligned}
$$

From the fact that the excess current component at $V=V_{p}$ and the tunnel current component at $V=V_{p r}\left(V_{p r}\right.$ is projected peak voltage) are negligible, it is reasonable to now set both these 


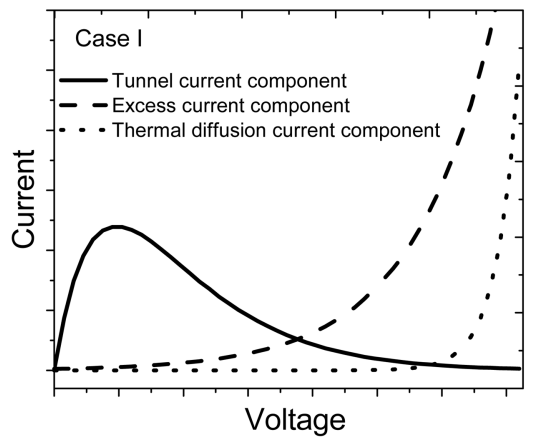

(a)

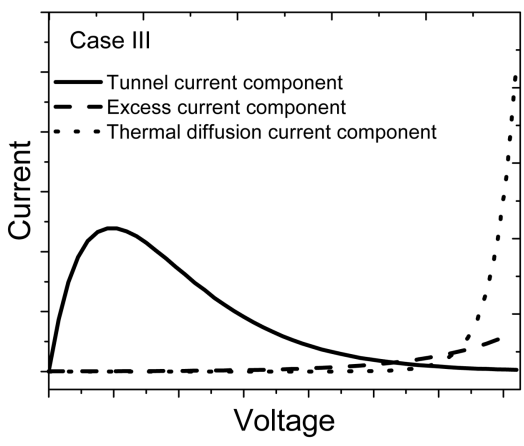

(c)

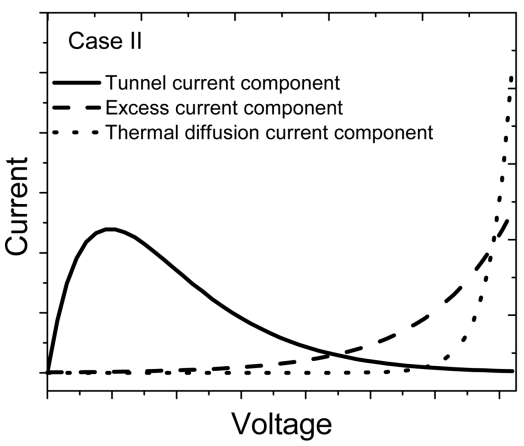

(b)

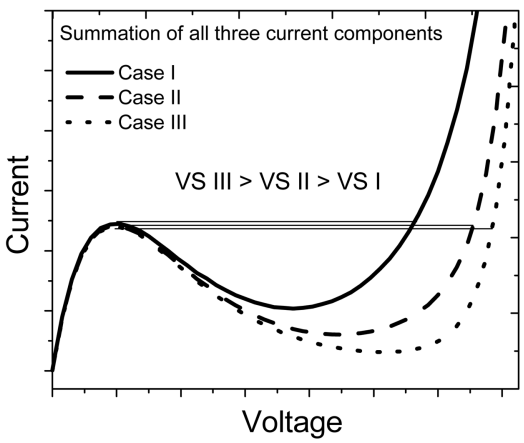

(d)

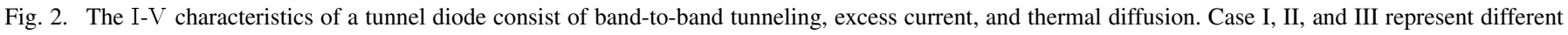

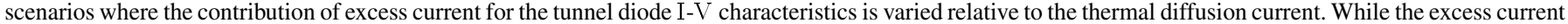

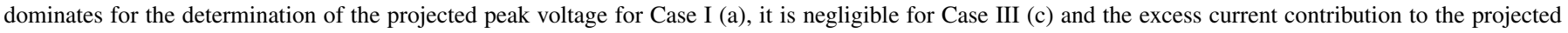
peak voltage is intermediate for (b).

terms to zero, then the voltage swing $V_{S W}$ can be more simply expressed as

$$
\begin{aligned}
V_{S W} & =V_{\mathrm{Pr}}-V_{P} \approx \frac{1}{A} \ln \frac{I_{P}}{I_{V}}+\left(V_{V}-V_{P}\right) \\
& =\left[\frac{1}{A} \ln (\mathrm{PVCR})\right]+\left(V_{V}-V_{P}\right) .
\end{aligned}
$$

The second term $V_{v}-V_{p}$ is assumed constant. This is a good approximation for the intrinsic RITD device characteristics. For instance, as the doping concentration is changed, the Fermi levels are scaled resulting in a shift of the peak and valley positions, which affect the overlap voltages. Therefore, not only will the peak voltage shift, but also the valley voltage will move by a similar scaled amount. For this approximation, no parasitic series resistance is assumed. From (3), the voltage swing is proportional to the logarithm of the $I_{p}$ and $I_{v}$ (or equivalently, the $J_{p}$ and $J_{v}$ ) quotient, i.e., PVCR.

\section{B. Role of $\delta$-Doping Levels of an RITD on its Voltage Swing}

Fig. 3 shows the $\delta$-doping carrier concentration versus measured voltage swing, PVCR, $J_{P}$, and $J_{V}$. The sheet carrier concentration was assumed to be distributed over a $1 \mathrm{~nm}$ wide box for the calculations. All devices shown in Fig. 3 were postgrowth annealed at $750{ }^{\circ} \mathrm{C}$ for $1 \mathrm{~min}$.

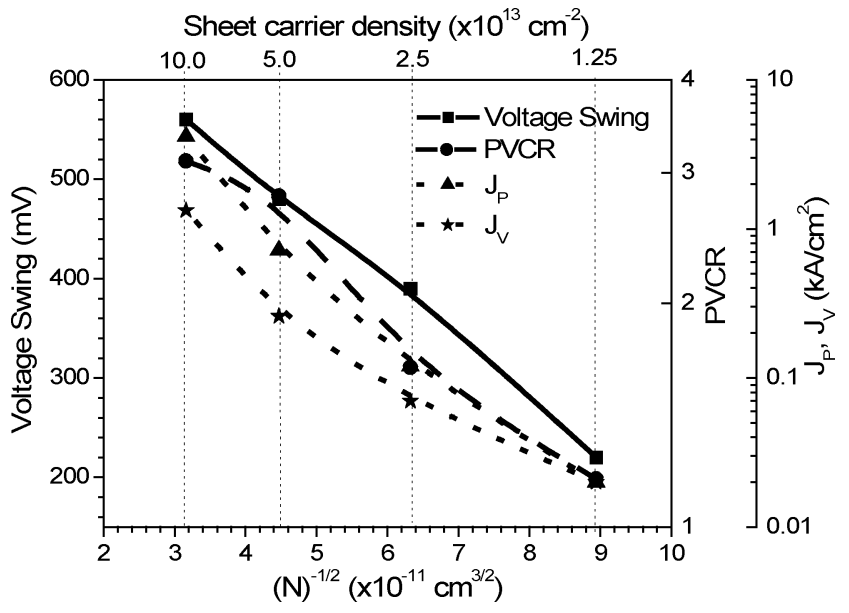

Fig. 3. Voltage swing, PVCR, $J_{P}$, and $J_{V}$ versus $\delta$-doping density showing a $N^{-1 / 2}$ dependence of voltage swing and $\exp \left(-N^{-1 / 2}\right)$ dependence of $J_{P}$, $J_{V}$, and PVCR on carrier concentration, where $N$ is an estimated bulk doping density assuming a $\delta$-doped waist that is $1 \mathrm{~nm}$ in lateral extent.

The interband tunneling probability $(P)$ [17], which determines the tunneling current, decreases exponentially with the depletion width $(W)$

$$
I_{P} \propto P=\exp \left\lfloor\frac{-\left(\pi \cdot W \cdot m^{* 1 / 2} E_{g}^{3 / 2}\right)}{\left(4 \sqrt{2} q \hbar \cdot\left(V_{b i}-V\right)\right)}\right\rfloor
$$


and $W$ is inversely proportional to the square root of the doping concentration

$$
W=\left[\frac{2 \varepsilon_{s}}{q}\left(\frac{2}{N}\right) \cdot\left(V_{b i}-V\right)\right]^{1 / 2}
$$

where $N=N_{A}=N_{D}$ in a symmetric p-n junction and $\varepsilon_{s}$ is the dielectric constant of Si. The exp $\left(-N^{-1 / 2}\right)$ dependence of the peak current density in these devices on the doping density was found to be in good agreement, as shown in Fig. 3, as evidenced by its slopes. It should be noted that the peak current in (4) is proportional to the maximum tunneling probability and, therefore, assumes the resonance tunneling condition is achieved regardless of the external bias necessary to bring the confined energy levels within the $\delta$-doped regions to be coincident or the confinement energy magnitude within the quantum wells.

The excess current is given by Chynoweth [18]

$$
\begin{aligned}
& I_{e x} \propto D_{x} \\
& \cdot \exp \left[\left(\frac{-\alpha_{x} \cdot W \cdot e^{0.5}}{2}\right) \times\left(E_{g}-e V+0.6 \cdot e \cdot\left(V_{n}-V_{p}\right)\right]\right.
\end{aligned}
$$

which has the same $\exp \left(-N^{-1 / 2}\right)$ dependence on doping density as the peak current from the depletion width, but with a different prefactor in the exponent. This dependence was observed in the relation between $J_{V}$ versus doping density, as shown in Fig. 3, which implies that the excess current likely plays a significant role in many $\mathrm{Si} / \mathrm{SiGe}$ RITDs. In (4)-(6), $m^{*}$ is the electron effective mass, $E_{g}$ is the bandgap, $D_{x}$ is the density of states of defects in the forbidden bandgap, $\hbar$ is the reduced Plank constant, $V_{n}$ and $V_{p}$ are the Fermi energy levels on the $n$ and $p$ side, respectively, and $\alpha_{x}$ is a constant containing a reduced effective mass.

It can be assumed that the defect density $D_{x}$ is similar amongst all the diodes plotted in Fig. 3, each having undergone identical thermal cycling during growth and annealing. Therefore, the key remaining difference amongst each of the RITDs presented in Fig. 3 rests on the doping density, embedded within the depletion width and its prefactor $a$ in the exponent of $\exp \left(-a \cdot N^{-1 / 2}\right)$ in $J_{p}$ and $J_{v}, \mathrm{PVCR}=J_{p} / J_{v}$ shows a dependence on the doping density in the same fashion as $J_{p}$ and $J_{v}$, with a prefactor in the exponent that is the difference between the two. As a result, the voltage swing shows a linear dependence on $N^{-1 / 2}$ according to (3). This linear dependence was also observed in Fig. 3.

\section{Correlation of Voltage Swing With Other Figure-of-Merits}

The attribution of the excess current component to voltage swing and its correlation to the other figure-of-merits was also investigated using different annealing temperatures for the RITD with $1 \times 10^{14} \mathrm{~cm}^{-2} \delta$-doping sheet carrier densities. As mentioned earlier, the main purpose of post-growth annealing is to reduce point defects created during LT-MBE growth which was adopted to suppress dopant segregation and outdiffusion from the $\delta$-doped layers. A secondary effect of post-growth annealing on peak current component (4) arises from the effectively broadened depletion width $\mathrm{W}$ due to outdiffusion from the $\delta$-doping spikes and interdiffusion and compensation within

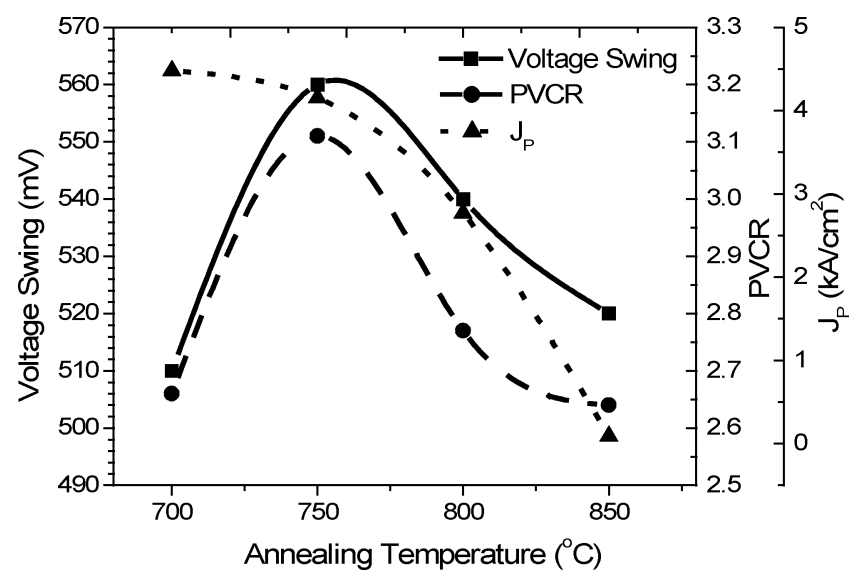

Fig. 4. Voltage swing, PVCR, $I_{P}$ versus annealing temperatures. The strong correlation is observed between PVCR and voltage swing. The low PCVR despite high $J_{P}$ at $700^{\circ} \mathrm{C}$ indicates that the defect density states in this RITD device elevates the excess current to dominate over the thermal diffusion current.

the intrinsic tunneling spacer in between [15]. This broadened depletion region causes a decrease of $J_{P}$ as annealing temperature increases, shown in Fig. 4. Two parameters of excess current component in (6), the defect density of states in the forbidden bandgap, $D_{x}$, and depletion width, $W$ are affected by post-growth annealing process.

From previous studies, the defect states inside the forbidden bandgap created during LT-MBE are mostly attributed to vacancies that have distinct energy levels [19] and these defect densities were observed to be reduced by post-growth heat treatments [20]. Shown in Fig. 4 are voltage swing, PVCR, and $J_{P}$ versus different post-growth RTA temperatures, $700{ }^{\circ} \mathrm{C}$, $750{ }^{\circ} \mathrm{C}, 800{ }^{\circ} \mathrm{C}$, and $850{ }^{\circ} \mathrm{C}$. A strong correlation between PVCR and voltage swing is observed here too. It is in good agreement with a previous study on tunneling spacer thickness [15]. However, despite the highest $J_{P}$ after the $700{ }^{\circ} \mathrm{C}$ anneal, the PVCR is low. This is due to the high excess current. The annealing at $700{ }^{\circ} \mathrm{C}$ was not high enough to appreciably reduce the density of vacancy defect states, $D_{x}$ which, up to an anneal temperature of $750{ }^{\circ} \mathrm{C}$, is a more important factor than the depletion-width-widening for the contribution to the excess current.

\section{CONCLUSION}

From the study of the effects of varying $\delta$-doping densities and varying post-growth annealing temperatures on voltage swing, the excess current I-V characteristics under forward bias dominates and determines the projected peak voltage (Case I in Fig. 2). The strong correlation of PVCR and voltage swing shown in both Figs. 3 and 4 suggests that devices with higher PVCR have smaller excess current components, therefore, their projected peak voltages are pushed closer to the regime where the thermal diffusion current is dominant, while a projected peak voltage in a lower PVCR device occurs in the range where the excess current component is dominant. Thus, it was found that the voltage swing of a tunnel diode, which determines the operational voltage range for circuit latching, should be maximized concurrently during PVCR optimization. 


\section{REFERENCES}

[1] J. P. A. van der Wagt, A. C. Seabaugh, and E. A. Beam, "RTD/HFET low standby power SRAM gain cell," IEEE Electron Device Lett., vol. 19, no. 1, pp. 7-9, Jan. 1998.

[2] P. Mazumder, S. Kulkarni, M. Bhattacharya, P. S. Jian, and G. I. Haddad, "Digital circuit applications of resonant tunneling devices," Proc. IEEE, vol. 86, pp. 664-686, 1998.

[3] K. Arai, H. Matsuzaki, K. Maezawa, T. Otsuji, and M. Yamamoto, "Static frequency divider featuring reduced circuit complexity by utilizing resonant tunneling diodes in combination with HEMTs," IEEE Electron Device Lett., vol. 18, no. 11, pp. 544-546, Nov. 1997.

[4] T. P. E. Broekaert, B. Brar, J. P. A. van der Wagt, C. Seabaugh, T. S. Moise, F. J. Morris, E. A. Beam, III, and G. A. Frazier, "A monolithic 4-bit 2-Gsps resonant tunneling analog-to-digital converter," IEEE J. Solid State Circuits, vol. 33, no. 9, pp. 1342-1349, Sep. 1998.

[5] T. A. Richard, E. I. Chen, A. R. Sugg, G. E. Höfler, and N. Holonyak, Jr., "High current density carbon-doped strained-layer GaAs $\left(p^{+}\right)$-In$\operatorname{GaAs}\left(n^{+}\right)-\operatorname{GaAs}\left(n^{+}\right) p-n$ tunnel diodes," Appl. Phys. Lett., vol. 63, pp. 3613-3615, 1993.

[6] S. L. Rommel, T. E. Dillon, M. W. Dashiell, H. Feng, J. Kolodzey, P. R. Berger, P. E. Thompson, K. D. Hobart, R. Lake, A. C. Seabaugh, G. Klimeck, and D. K. Blanks, "Room temperature operation of epitaxially grown $\mathrm{Si} / \mathrm{Si}_{0.5} \mathrm{Ge}_{0.5} / \mathrm{Si}$ resonant interband tunneling diodes," Appl. Phys. Lett., vol. 73, pp. 2191-2193, Oct. 1998.

[7] N. Jin, S.-Y. Chung, A. T. Rice, P. R. Berger, P. E. Thompson, C. Rivas, R. Lake, S. Sudirgo, J. J. Kempisty, B. Curanovic, S. L. Rommel, K. D. Hirschman, S. K. Kurinec, P. H. Chi, and D. S. Simons, "Diffusion barrier cladding in $\mathrm{Si} / \mathrm{SiGe}$ RITDs and their patterned growth on PMOS source/drain regions," IEEE Trans. Electron Devices, Special Issue "Nanoelectronics", vol. 50, pp. 1876-1884, 2003.

[8] S.-Y. Chung, R. Yu, N. Jin, S.-Y. Park, P. R. Berger, and P. E. Thompson, "Si/SiGe resonant interband tunnel diode with $f_{r 0} 20.2$ $\mathrm{GHz}$ and peak current density $218 \mathrm{kA} / \mathrm{cm}^{2}$ for K-band mixed-signal applications," IEEE Electron Device Lett., vol. 27, pp. 364-367, May 2006.

[9] R. Duschl and K. Eberl, "Physics and applications of Si/SiGe/Si resonant interband tunneling diodes," Thin Solid Films, vol. 380, pp. $151-153,2000$.

[10] D. J. Paul, P. See, I. V. Zozoulenko, K.-F. Berggren, B. Kabius, B. Hollander, and S. Mantl, "Si/SiGe electron resonant tunneling diodes," Appl. Phys. Lett., vol. 77, pp. 1653-1655, 2000.

[11] S. Sudirgo, R. P. Nandgaonkar, B. Curanovic, J. L. Hebding, R. L. Saxer, S. S. Islam, K. D. Hirschman, S. L. Rommel, S. K. Kurinec, P. E. Thompson, N. Jin, and P. R. Berger, "Monolithically integrated $\mathrm{Si} / \mathrm{SiGe}$ resonant interband tunnel diode/CMOS demonstrating low voltage MOBILE operation," Solid State Electron., vol. 48, pp. 1907-1910, Oct./Nov. 2004.

[12] S.-Y. Chung, N. Jin, P. R. Berger, R. Yu, P. E. Thompson, R. Lake, S. L. Rommel, and S. K. Kurinec, "3-terminal Si-based negative differential resistance circuit element with adjustable peak-to-valley current ratios using a monolithic vertical integration," Appl. Phys. Lett., vol. 84, pp. 2688-2690, Apr. 2004.

[13] R. W. Keyes, "Physics of digital devices," Rev. Mod. Phys., vol. 61, pp. 279-287, 1989.

[14] H. Zhang, P. Mazumder, L. Ding, and K. Yang, "Performance modeling of resonant tunneling-based random-access memories," IEEE Trans. Nanotech., vol. 4, no. 4, p. 472, Jul. 2005.

[15] N. Jin, S.-Y. Chung, R. Yu, R. M. Heyns, P. R. Berger, and P. E. Thompson, "The effect of spacer thickness on Si-based resonant interband tunneling diode performance and their application to low-power tunneling diode SRAM circuits," IEEE Trans. Electron Devices, vol. 53, pp. 2243-2249, Sep. 2006.

[16] S. M. Sze, Physics of Semiconductor Devices, 2nd ed. New York: Wiley, 1981, p. 529.

[17] - Physics of Semiconductor Devices, 2nd ed. New York: Wiley, 1981, p. 522.

[18] A. G. Chynoweth, W. L. Feldmann, and R. A. Logan, "Excess tunnel current in silicon Esaki junctions," Phys. Rev., vol. 121, pp. 684-694, 1961.

[19] S.-Y. Chung, N. Jin, A. T. Rice, P. R. Berger, R. Yu, Z.-Q. Fang, and P. E. Thompson, "Growth temperature and dopant species effects on deep levels in Si grown by low temperature molecular beam epitaxy," J. Appl. Phys., vol. 93, pp. 9104-9110, Jun. 2003.
[20] S.-Y. Chung, N. Jin, R. E. Pavlovicz, P. R. Berger, R. Yu, Z.-Q. Fang, and P. E. Thompson, "Annealing effect on defects in Si grown by low temperature molecular beam epitaxy and its attribution to the peak and excess currents in Si-based tunnel diodes," J. Appl. Phys., vol. 96, pp. 747-753, Jul. 2004.

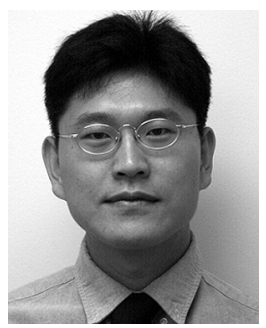

Sung-Yong Chung (S'00) received the B.S. degree in metallurgical engineering from Korea University, Seoul, in 1999, and the M.S. and Ph.D. degrees in electrical engineering from The Ohio State University, Columbus, in 2002 and 2005, respectively.

Since Fall 2005, he has been a staff member at Spansion, Inc., Sunnyvale, CA, a recent spin-off from AMD, working on flash memory. He has coauthored over 17 archival refereed journal articles and has three U.S. patents pending.

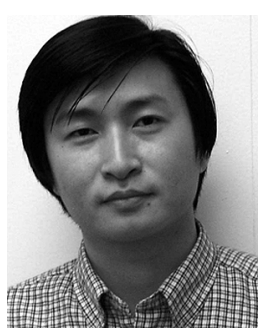

Niu Jin (S'03-M'04) received both the B.E. and M.E. degrees in materials science and engineering from the Hefei University of Technology, Hefei, China, in 1995 and 1998, respectively, and the M.S. and Ph.D. degrees in electrical engineering from The Ohio State University, Columbus, in 2001 and 2004, respectively, where he worked on Si-based tunneling devices.

Currently, he is a Postdoctoral Research Associate at the University of Illinois, Urbana-Champaign, Urbana, where he works on nanofluidic devices. He has coauthored over 18 archival refereed journal articles and has two U.S. patents pending.

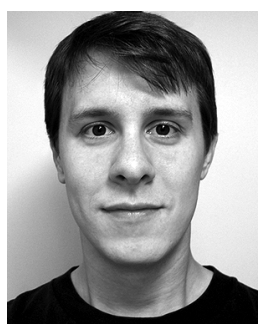

Ryan E. Pavlovicz received the B.S. degree in electrical engineering from The Ohio State University, Columbus, in 2004. He is currently working towards the $\mathrm{Ph} . \mathrm{D}$. degree in biomedical engineering at The Ohio State University. His graduate work focuses on computational biochemistry with applications for drug design and protein engineering.

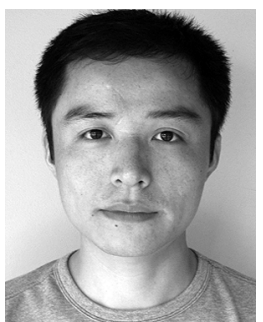

Ronghua Yu received the B.S. degree in physics from the University of Electronic Science and Technology of China, Chengdu, China, in 1994, and the M.S. degree in physics from the University of Science and Technology of China, Hefei, Anhui, China, in 1997. He is currently working towards the Ph.D. degree at The Ohio State University, Columbus

His research interests include semiconductor physics, and the physics and technology of nanometer-scaled materials and structures. His current research activities are centered around $\mathrm{Si} / \mathrm{SiGe-based} \mathrm{resonant} \mathrm{interband}$ tunnel diodes and related structures. 


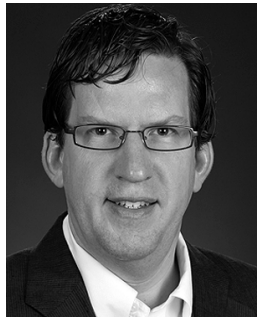

Paul R. Berger (S'84-M'91-SM'97) received the B.S.E. degree in engineering physics, and the M.S.E. and $\mathrm{Ph} . \mathrm{D}$. degrees in electrical engineering from the University of Michigan, Ann Arbor, in 1985, 1987, and 1990, respectively.

$\mathrm{He}$ is currently a Professor in both the Electrical and Computer Engineering Department and the Physics Department, The Ohio State University, Columbus. He is the Founder and Director of Ohio's new Nanoscale Patterning Laboratory and Director of the Nanofabrication and Materials Processing Center (NanoMPC). Currently, he is actively working on $\mathrm{Si} / \mathrm{SiGe}$ nanoelectronic devices and fabrication processes, Si-based resonant interband tunneling diodes and quantum functional circuitry, conjugated polymer-based optoelectronic and electronic devices, molecular electronics, and semiconductor materials, fabrication and growth. From 1985 to 1990, he worked on III-V compound molecular beam epitaxy, InGaAs/GaAs strained layer growth and self-assembled quantum dots, and optoelectronic devices and integrated circuits. From 1990 to 1992, he worked at Bell Laboratories, Murray Hill, NJ, on optoelectronic devices and OEICs. He was an Assistant Professor (1992-1997) and an Associate Professor (1997-2000) at the University of Delaware in Electrical and Computer Engineering. In 1999, a sabbatical leave lead him to work at the Max-Planck Institute for Polymer Research, Mainz, Germany, and Cambridge Display Technology, Ltd., Cambridge, U.K. In 2000, he joined The Ohio State University as an Associate Professor. He has coauthored nearly 80 archival refereed journal articles, about 75 invited and regular conference presentations, three book sections and has been issued ten patents with four more pending.

Dr. Berger is a recipient of the National Science Foundation's 1996 Faculty Early Career Development Award. In 1998, he received the Defense Advanced Research Projects Agency (DARPA) ULTRA Sustained Excellence Award and the 2006 OSU COE Lumley Research Award. He was a co-organizer of the SRC sponsored Si Tunnel Diode and CMOS/HBT Integration Workshop in Washington, DC. He has been on the Program and Advisory Committees of the International Electron Devices Meeting, International Semiconductor Device Research Symposium, Electronic and Photonic Materials, Devices and Systems, and SPIE Conferences. He currently is the Chair of the Columbus EDS/LEOS Chapter and Faculty Advisor to the Ohio State IEEE Student Chapter. He is a member of the Optical Society of America, Materials Research Society, and American Society for Engineering Education.

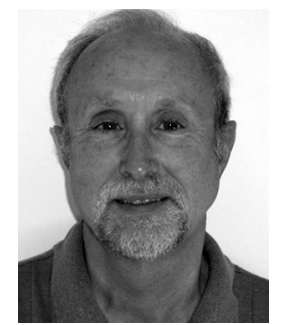

Phillip E. Thompson (SM'00) received the Ph.D. degree in physics from the University of Delaware, Newark, in 1975.

He is Head of the Si MBE Growth and Characterization Section, Electronics Science and Technology Division, Naval Research Laboratory (NRL), Washington, DC. He joined NRL in 1981 as a Research Physicist. He has over 25 years experience in electronic materials, including 18 years in MBE growth. He has over 200 publications and presentations. He holds five patents and has four pending.

Dr. Thompson received two NRL Tech Transfer Awards and the Defense Advanced Research Projects Agency (DARPA) ULTRA Program Sustained Excellence Award in 1998. He is a member of the American Physical Society (APS) and MRS. 